\title{
Índices de competição em nível de árvore individual para espécies da floresta ombrófila mista
}

\section{Competition indices at the individual tree level in a mixed ombrophylous forest}

\author{
Cristine Tagliapietra Schons ${ }^{1}$ (D), Afonso Figueiredo Filho ${ }^{1,2}$ (D), Tomaz Longhi Santos ${ }^{1}$ (D), \\ Rodrigo Geroni Mendes Nascimento ${ }^{3}$ (1) \\ ${ }^{1}$ Universidade Federal do Paraná - UFPR, Curitiba, PR, Brasil \\ ${ }^{2}$ Universidade Estadual do Centro-Oeste - UNICENTRO, Irati, PR, Brasil \\ ${ }^{3}$ Universidade Federal Rural da Amazônia - UFRA, Belém, PA, Brasil
}

\begin{abstract}
Como citar: Schons, C. T., Figueiredo Filho, A., Longhi-Santos, T., \& Nascimento, R. G. M. (2020). Índices de competição em nível de árvore individual para espécies da floresta ombrófila mista. Scientia Forestalis, 48(128), e3275. https://doi.org/10.18671/scifor.v48n128.13
\end{abstract}

\begin{abstract}
Resumo
O objetivo deste estudo foi investigar interações competitivas que influenciam o crescimento em área transversal para árvores individuais de quatro espécies em uma Floresta Ombrófila Mista (FOM) Montana no sul do Brasil, buscando identificar um índice que melhor represente a competição para essas espécies. Os dados deste estudo provieram de parcelas permanentes instaladas e monitoradas desde o ano de 2002 na Floresta Nacional de Irati (FLONA de Irati), estado do Paraná, Brasil. Foram avaliados 7 índices de competição independentes da distância, com base em análise gráfica e no coeficiente de correlação linear entre cada índice e o incremento periódico anual em área transversal. $O$ índice de competição de melhor desempenho foi selecionado para predizer taxas de crescimento em função do índice de competição das espécies Araucaria angustifolia, Cedrela fissilis, Ocotea puberula e Ocotea odorifera e analisar a dinâmica da competição. De maneira geral, todos os índices avaliados apresentaram boa correlação com o crescimento de árvores individuais, com destaque para os índices Lorimer, Glover e Holl e Stage. A relação competição-crescimento apresentou diferenças entre as espécies, sugerindo que há espécies com crescimento mais e menos sensíveis à variação da competição, provavelmente relacionado às suas características ecológicas. Os índices de competição independentes da distância testados são recomendados para representar a competição das espécies estudadas em Floresta Ombrófila Mista e contribuem com subsídios no contexto de submodelos de incremento na modelagem em nível de árvore individual.
\end{abstract}

Palavras-chave: Índices independentes da distância; Incremento periódico; Floresta com Araucária; Manejo florestal.

\begin{abstract}
This study aimed to investigate competitive interactions which influence the growth in individual basal area for individual trees of four species in a Montane Mixed Ombrophilous Forest (FOM) in southern Brazil, seeking to identify an index that best represents the competition for these species. The data came from permanent plots installed and monitored since 2002 in the Irati National Forest (FLONA de Irati), Paraná state, Brazil. Seven distance-independent competition indices were evaluated, based on graphical analysis and the linear correlation coefficient between each index and the periodic annual increment in individual basal area. The best performance competition index was selected to predict growth rates related to competition indices of the species Araucaria angustifolia, Cedrela fissilis, Ocotea puberula and Ocotea odorifera and analyze the dynamics of competition. In general, all indices evaluated showed a good correlation with the growth of individual trees, especially for the Lorimer, Glover and Holl and Stage indices. The competition-growth relationship showed differences between species, suggesting that there
\end{abstract}

Fonte de financiamento: Coordenação de Aperfeiçoamento de Pessoal de Nível Superior (CAPES) - Código de Financiamento 001.

Conflito de interesse: Nada a declarar.

Autor correspondente: cristschons@gmail.com

Recebido: 6 fevereiro 2019.

Aceito: 7 novembro 2019.

Editor: Paulo Henrique Müller Silva.

(c) (i) Este é um artigo publicado em acesso aberto (Open Access) sob a licença Creative Commons Attribution, que permite uso, distribuição e

cc) reprodução em qualquer meio, sem restrições desde que o trabalho original seja corretamente citado. 
are species with more and less sensitive growth to the competition variation, probably related to their ecological characteristics. The distance-independent competition indices tested in this study are recommended to represent competition in Mixed Ombrophilous Forest and contribute with subsidies in the context of increment sub-models in individual tree level modeling.

Keywords: Distance-independent indices; Periodic increment; Araucaria forest; Forest management.

\section{INTRODUÇÃO}

A competição, em ambientes florestais, pode ser definida como uma interação entre árvores causada pela concorrência por recursos em quantidade limitada, o que acarreta redução da sobrevivência, crescimento e/ou a reprodução do indivíduo em questão (Begon et al., 2007). É, portanto, um processo ecológico fundamental que desempenha importante papel na dinâmica populacional, sobrevivência, crescimento e substituição de espécies (Peet \& Christensen, 1987). Tomé \& Burkhart (1989) identificaram três componentes primários da competição: (1) o ambiente geral da árvore; (2) influências microambientais e genéticas; e (3) a influência dos vizinhos locais.

Além disso, existem diferentes tipos ou formas de competição. Uma das distinções mais importantes é o modo de competição, que pode ser unilateral (one-sided competition) e bilateral (two-sided competition) (Zhao et al., 2004). Unilateral refere-se à competição entre árvores por luz, em que as árvores maiores não são afetadas por árvores vizinhas menores, enquanto que a competição bilateral representa a concorrência por água e nutrientes, considerando que os recursos são concorridos (igualmente, ou proporcionais ao tamanho) por todas as árvores (Burkhart \& Tomé, 2012). Também é possível se referir à competição como intraespecífica, entre indivíduos da mesma espécie, e como interespecífica, quando ocorre entre indivíduos de espécies diferentes (Weiskittel et al., 2011).

Uma maneira de representar a competição é por meio do uso de índices de competição. Além de expressarem o estresse competitivo de cada indivíduo da floresta, eles constituem o centro de todo modelo de crescimento de árvore individual, onde são utilizados para estimar o crescimento e/ou mortalidade durante uma simulação (Téo et al., 2015). Munro (1974) classificou os índices de competição como independentes de distância (não exigindo localização de árvores individuais) e dependentes de distância (exigindo coordenadas de localização das árvores para computação). Posteriormente, uma nova classe de medidas de competição foi apresentada, denominando índices "semi-independentes de distância" (Burkhart \& Tomé, 2012).

Apesar dos avanços obtidos em florestas heterogêneas, melhorar a compreensão a respeito do comportamento da competição em um ambiente natural e de como ela condiciona o crescimento individual das árvores é necessária, visando projetar o desenvolvimento dessas florestas. Ademais, não há um consenso sobre a superioridade de algum índice de competição, uma vez que a habilidade preditiva varia conforme as condições da floresta em estudo (Curto, 2015) e as espécies (Tonini, 2007). As evidências também são contraditórias quanto à melhoria de predições de crescimento com a inclusão de efeitos espaciais na descrição do ambiente competitivo das árvores (Peltoniemi \& Mäkipää; 2011). Ambos os índices, dependentes e independentes da distância, têm sido aplicados em florestas mistas, no entanto, o mapeamento das árvores é bastante dispendioso e oneroso (Weiskittel et al., 2011) e alguns estudos comparativos concluíram que os índices independentes da distância são tão eficazes quanto os dependentes da distância (Lorimer, 1983; Orellana, 2014).

Neste contexto, o objetivo deste estudo foi investigar interações competitivas que influenciam o crescimento de árvores individuais de quatro espécies em um remanescente de Floresta Ombrófila Mista (FOM), buscando identificar um índice que melhor represente a competição para essas espécies. As seguintes questões foram levantadas: (i) Interações competitivas em uma Floresta Ombrófila Mista podem ser representadas por meio de um índice de competição independente da distância?; (ii) As respostas de crescimento à competição são equivalentes entre as espécies? 


\section{Área de estudo e obtenção dos dados}

O local de estudo foi um fragmento da Floresta Ombrófila Mista com 1273 ha, livre de intervenções há mais de 70 anos na Floresta Nacional de Irati (FLONA de Irati), estado do Paraná, Brasil. A FLONA de Irati está situada no segundo planalto paranaense, no âmbito dos municípios de Fernandes Pinheiro e Teixeira Soares (25²7'56" S, 50³7'51" O), a uma altitude média de 885 m. Segundo a classificação de Koppen, a região apresenta clima do tipo Cfb Subtropical Úmido Mesotérmico (Alvares et al., 2013), caracterizado por verões frescos, geadas severas e frequentes e sem estação seca, com precipitação média anual de $1.442 \mathrm{~mm}$. O limiar médio das temperaturas de Irati varia aproximadamente entre 13 e $23,5^{\circ} \mathrm{C}$.

Na área, 25 blocos de 1 ha subdivididos em 100 parcelas permanentes de $50 \times 50 \mathrm{~m}$ foram instalados no ano de 2002. Todas as árvores com DAP igual ou superior a $10 \mathrm{~cm}$ têm sido remedidas a cada 3 anos (2002, 2005, 2008, 2011, 2014 e 2017), totalizando uma amostra de mais de 14.000 árvores (Figueiredo Filho et al., 2010).

As espécies Araucaria angustifolia (Araucária), Cedrela fissilis (Cedro), Ocotea puberula (Canela-guaicá) e Ocotea odorifera (Sassafrás) foram selecionadas para este estudo vislumbrando desenvolvimento de planos de manejo específicos no futuro, atendendo a alguns critérios: maior Índice de Valor de Importância (IVI); boa distribuição ao longo da área das parcelas permanentes; potencial econômico da espécie. O IVI foi calculado para avaliação das espécies, bem como outros estimadores fitossociológicos como a densidade, dominância e frequência, permitindo também ter-se uma ideia da biodiversidade da comunidade. Os valores relativos e absolutos destas variáveis foram calculados e analisados considerando a área amostrada (25 ha), sendo a frequência obtida por parcela $(50 \mathrm{~m}$ x $50 \mathrm{~m}$ ), para o ano de medição de 2017. Os cálculos foram realizados utilizando-se do suplemento FlorExel (Arce et al., 2002), computadorizado para Microsoft Excel®.

\section{Estrutura diamétrica}

Na sequência, foi realizada a análise da distribuição diamétrica considerando valores de frequência de árvores obtidos a partir dos dados das parcelas permanentes. Considerou-se a frequência de indivíduos em classes de amplitude de $10 \mathrm{~cm}$ de diâmetro (FigueiredoFilho et al., 2010), para o ano de 2002 e 2017.

\section{Índices de competição}

Visando representar a competição, o nível de competição experimentado de cada árvore individual abrangida pelas parcelas permanentes foi quantificado testando diferentes índices de competição independentes da distância listados na Tabela 1. Todas as árvores foram incluídas na análise, ora funcionando como árvore objetivo, ora como árvores competidoras. As competidoras à árvore-objetivo foram consideradas como sendo as demais árvores integrantes da parcela. Árvore-objetivo é aquela para a qual se está calculando o índice de competição. Parcelas de tamanho 50 × 50 m (2500 m²) foram tomadas para os cálculos, pois conforme identificado por Chassot et al. (2011) e Orellana (2014) esse foi o tamanho que apresentou maiores correlações com o crescimento e significância $(p<0,05)$ no ajuste.

Os sete índices independentes de distância selecionados têm sido amplamente utilizados e suas interpretações estão inteiramente relacionadas à maneira como são calculados. IC1, IC2 e IC7, por assumirem como competidoras árvores com diâmetro maior que a árvore objetivo, são índices que representam a competição unilateral (luz), enquanto os demais índices representam a competição bilateral. 
TABELA 1 - Índices de competição independentes da distância testados para um fragmento de Floresta Ombrófila Mista, FLONA de Irati, PR.

\begin{tabular}{|c|c|c|c|}
\hline Índice & Fórmula & Fonte & Interpretação \\
\hline $\begin{array}{l}\text { BAL (Basal Area } \\
\text { for Larger trees) }\end{array}$ & $\begin{array}{c}I C_{I}=\sum_{j=I}^{n j} G j \\
I C_{2}=\sum_{j=I}^{n j} G j / d_{i}\end{array}$ & $\begin{array}{l}\text { Stage (1973) } \\
\text { Lhotka \& Loewenstein } \\
\text { (2011) }\end{array}$ & $\begin{array}{c}\text { Quanto menor o índice, } \\
\text { menor é a competição sobre } \\
\text { a árvore } i \text {. }\end{array}$ \\
\hline Glover e Holl & $\begin{array}{c}I C_{3}=d_{i}^{2} / \bar{d}_{j}^{2} \\
C_{4}=d_{i}^{2} /\left(\sum_{j=1}^{n} d_{j}^{2}\right) / n\end{array}$ & $\begin{array}{l}\text { Glover \& Hool (1979) } \\
\text { Daniels et al. (1986) }\end{array}$ & $\begin{array}{c}\text { Quanto menor o índice, } \\
\text { maior é a competição sobre a } \\
\text { árvore } i \text {. }\end{array}$ \\
\hline Stage & $I C_{5}=d_{i} / d g$ & Stage (1973) & $\begin{array}{c}\text { Quanto menor o índice, } \\
\text { maior é a competição sobre a } \\
\text { árvore } i \text {. }\end{array}$ \\
\hline Lorimer & $I C_{6}=\sum_{j=1}^{n} d_{j} / d_{i}$ & Lorimer (1983) & $\begin{array}{c}\text { Quanto menor o índice, } \\
\text { menor é a competição sobre } \\
\text { a árvore } i \text {. }\end{array}$ \\
\hline $\begin{array}{l}\text { Tomé e } \\
\text { Burkhart }\end{array}$ & $I C_{7}=d_{i} / d_{\text {máx }}$ & Tomé \& Burkhart (1989) & $\begin{array}{c}\text { Quanto menor o índice, } \\
\text { maior é a competição sobre a } \\
\text { árvore } i \text {. }\end{array}$ \\
\hline
\end{tabular}

n: número de árvores competidoras; Gj: área transversal das árvores competidoras $\left(\mathrm{m}^{2} / \mathrm{ha}\right)$ com dap maior que o dap da árvore central; di: DAP da árvore objetivo (cm); dj: DAP das árvores competidoras (cm); dj: DAP médio das árvores competidoras $(\mathrm{cm})$; dg: diâmetro médio quadrático $(\mathrm{cm})$; dmáx: DAP da árvore mais grossa; i: número de ordem da árvore central considerada (árvore objetivo); j: número de ordem da árvore competidora.

Os índices BAL (Basal Area for Larger trees) (IC1 e IC2) atingem o valor mínimo de competição quando não há nenhuma árvore competidora maior que a árvore objetivo $i$ na parcela. À medida que o número de árvores que possuem diâmetro maior que a árvore objetivo $i$ aumenta, maior é a competição. A relação verificada para o índice de Lorimer (IC6) é similar, considerando que quanto menor o valor do índice, menor a competição, sendo este afetado pelo número de árvores competidoras.

De maneira contrária, os índices Glover e Holl (IC3 e IC4), Stage (IC5) e Tomé e Burkhart (IC7) sugerem que quando seu valor está próximo de zero a competição é máxima, ou seja, quanto mais o diâmetro da árvore $i$ se distância, para menos, do diâmetro médio, do diâmetro quadrático ou do diâmetro máximo da parcela, respectivamente, menor é o valor do índice e maior é a competição sobre a árvore $i$. No caso de IC7, o índice assume valor máximo de 1 na ocasião de a árvore-objeto $i$ ser aquela de diâmetro máximo da parcela.

\section{Avaliação dos índices de competição}

A avaliação dos índices de competição foi feita por meio da análise gráfica da relação entre os índices e o incremento periódico anual em área transversal (IPAg) entre as medições de 2002 e 2017 e do cálculo do coeficiente de correlação de Pearson (Daniels et al., 1986; Chassot et al., 2011; Castro et al., 2014; Tenzin et al., 2017). Foram calculadas correlações considerando todas as espécies em conjunto (geral) e para cada uma das espécies selecionadas. 
$I P A g=\frac{g 2017-g 2002}{n}$

Em que: $I P A g=$ incremento periódico anual em área transversal $\left(\mathrm{m}^{2} \text {.ano-1 }\right)^{-1}$ g2017 = área transversal da árvore em $2017\left(\mathrm{~m}^{2}\right) ;$ g2002 = área transversal da árvore em $2002\left(\mathrm{~m}^{2}\right) ; \mathrm{n}=$ intervalo entre as medições (anos).

Para a correlação o valor logarítmico dos índices de competição e do incremento foi utilizado, buscando aproximar a relação na forma linear.

\section{Análise da competição}

Depois de selecionar o melhor índice de competição na área de estudo, para analisar as respostas de crescimento à competição para cada uma das espécies, o seguinte modelo dendrométrico não linear foi ajustado, com base em análises prévias (Castagneri et al., 2008), predizendo-se taxas de crescimento em função do índice de competição:

$I P A g=\beta_{0} \cdot \beta_{1}{ }^{I C}+\varepsilon_{i}$

Em que: IPAg = Incremento periódico em área transversal $\left(\mathrm{m}^{2}\right.$.ano $\left.{ }^{-1}\right)$; IC = Índice de competição; $\beta_{0}$ e $\beta_{1}=$ parâmetros do modelo; $\varepsilon_{i}=$ erro aleatório.

$\mathrm{O}$ ajuste do modelo não-linear foi realizado por meio do software $\mathrm{R}$, utilizando o algoritmo de Gauss-Newton. A precisão do modelo ajustado para cada espécie foi avaliada em relação ao valor do coeficiente de determinação $\left(R^{2}\right)$ e do erro padrão de estimativa em porcentagem (Syx\%).

\section{RESULTADOS E DISCUSSÃO}

\section{Estrutura horizontal, distribuição diamétrica e incremento periódico}

De um total de 136 espécies catalogadas na área, os resultados da análise da estrutura horizontal para o ano de 2017 das 20 espécies de maior Índice de Valor de Importância (IVI) estão listados em ordem decrescente na Tabela 2.

As espécies A. angustifolia, O. odorifera, C. fissilis e O. puberula, alvo deste estudo, destacam-se entre as espécie mais importantes ecologicamente na área, alcançando altos valores de IVI e também boa distribuição dentre as parcelas. A dominância do dossel por $A$. angustifolia fica caracterizada pelo valor de IVI de 36,77, dominância de 8,69 e densidade 42,04. O. odorifera, apesar de contar com o maior número de indivíduos dentre todas as espécies, conta com muitas árvores de diâmetros pequenos (IVI de 16,82, dominância de 1,78, densidade de 54,04).

As distribuições diamétricas para as espécies amostradas estão apresentadas na Figura 1. O. odorifera é a espécie que conta com uma distribuição caracteristicamente decrescente, conhecida como uma estrutura com curva exponencial negativa ("J" invertido), com poucas árvores alcançando diâmetros maiores que $40 \mathrm{~cm}$. As espécies $C$. fissilis e 0 . puberula apresentam distribuições unimodais assimétricas e muito semelhantes entre si. A espécie $A$. angustifolia está representada pela distribuição bimodal, com indivíduos melhor distribuídos entre as classes e alcançando maiores dimensões. Segundo Figueiredo Filho et al. (2010), a ocorrência de mais de uma moda para A. angustifolia pode indicar a possibilidade de ter ocorrido, no passado, cortes seletivos da espécie em algumas classes de diâmetro. Além disso, essa observação também pode estar relacionada à teoria de coortes (Ryder, 1965), a qual possui relação à origem das distribuições multimodais, indicando que distúrbios ocorrentes na floresta podem influenciar o recrutamento de um grupo de árvores em um mesmo intervalo de tempo (Ebling \& Péllico Netto, 2015). 
De modo geral, são pequenas as variações da distribuição dos índividuos de $A$. angustifolia e O. odorifera entre 2002 e 2017. No entanto, é possível perceber que para C. fissilis e 0 . puberula há um avanço dos diâmetros, com a diminuição da frequência de indivíduos de menor porte. $C$. fissilis e $O$. puberula são espécies caracterizadas dentro do grupo ecológico das pioneiras/secundárias iniciais, sendo que esse avanço pode ser um indício de regeneração deficiente. $\mathrm{O}$ número total de árvores abrangidas nas parcelas permanentes aumentou de 2002 para 2017, passando de 14519 para 14716.

No período de 15 anos (2002-2017) o maior IPAg médio identificado entre as espécies selecionadas foi o de $A$. angustifolia $\left(0,0030 \mathrm{~m}^{2}\right.$.ano-1 $)$, e o menor foi encontrado para a espécie O. odorifera $\left(0,0004 \mathrm{~m}^{2}\right.$.ano-1 $)$. C. fissilis e 0 . puberula apresentaram valores médios de 0,0020 e $0,0016 \mathrm{~m}^{2}$.ano-1. O IPAg médio geral contabilizando todas as espécies abrangidas pelas parcelas permanentes foi de $0,0010 \mathrm{~m}^{2}$.ano-1, com coeficiente de variação (CV) de 127,66\%, refletindo a grande variabilidade.

TABELA 2: Estimadores fitossociológicos da estrutura horizontal para o ano de 2017 do fragmento de Floresta Ombrófila Mista, FLONA de Irati, PR.

\begin{tabular}{|c|c|c|c|c|c|c|c|c|}
\hline Espécie & $\mathbf{N}$ & DA & DoA & FA & DR & DoR & $\mathbf{F R}$ & IVI \\
\hline & & $\underset{1}{\text { N.ha }}$ & $\mathrm{m}_{1}^{2}$ ha & $\%$ & $\%$ & $\%$ & $\%$ & $\%$ \\
\hline Araucaria angustifolia (Bertol.) Kuntze & 1051 & 42,04 & 8,69 & 99,00 & 7,14 & 26,97 & 2,66 & 36,77 \\
\hline Ocotea odorifera (Vell.) Rohwer & 1351 & 54,04 & 1,78 & 79,00 & 9,18 & 5,52 & 2,12 & 16,82 \\
\hline Ilex paraguariensis A. St.-Hil. & 1318 & 52,72 & 1,67 & 100,00 & 8,96 & 5,18 & 2,69 & 16,82 \\
\hline Ocotea porosa (Nees \& Mart.) Barroso & 479 & 19,16 & 3,02 & 75,00 & 3,25 & 9,38 & 2,02 & 14,65 \\
\hline Nectandra grandiflora Nees & 753 & 30,12 & 1,71 & 97,00 & 5,12 & 5,32 & 2,61 & 13,05 \\
\hline Cedrela fissilis Vell. & 371 & 14,84 & 1,68 & 92,00 & 2,52 & 5,23 & 2,47 & 10,22 \\
\hline Casearia decandra Jacq. & 748 & 29,92 & 0,78 & 98,00 & 5,08 & 2,41 & 2,63 & 10,13 \\
\hline Ocotea puberula (Rich.) Nees & 287 & 11,48 & 1,39 & 87,00 & 1,95 & 4,31 & 2,34 & 8,59 \\
\hline Nectandra megapotamica (Spreng.) Mez & 422 & 16,88 & 0,98 & 91,00 & 2,87 & 3,04 & 2,45 & 8,35 \\
\hline Coussarea contracta (Walp.) Müll. Arg. & 651 & 26,04 & 0,42 & 81,00 & 4,42 & 1,31 & 2,18 & 7,91 \\
\hline Syagrus romanzoffiana (Cham.) Glassman & 438 & 17,52 & 0,76 & 90,00 & 2,98 & 2,36 & 2,42 & 7,76 \\
\hline Matayba elaeagnoides Radlk. & 330 & 13,20 & 1,03 & 79,00 & 2,24 & 3,21 & 2,12 & 7,58 \\
\hline Ocotea diospyrifolia (Meisn.) Mez & 222 & 8,88 & 0,82 & 79,00 & 1,51 & 2,55 & 2,12 & 6,18 \\
\hline Casearia sylvestris Sw. & 396 & 15,84 & 0,40 & 74,00 & 2,69 & 1,25 & 1,99 & 5,93 \\
\hline Cinnamodendron dinisii Schwacke & 278 & 11,12 & 0,60 & 74,00 & 1,89 & 1,87 & 1,99 & 5,74 \\
\hline Myrcia hebepetala DC. & 381 & 15,24 & 0,25 & 88,00 & 2,59 & 0,76 & 2,36 & 5,72 \\
\hline Myrsine umbellata Mart. & 361 & 14,44 & 0,35 & 81,00 & 2,45 & 1,08 & 2,18 & 5,71 \\
\hline $\begin{array}{c}\text { Myrciaria floribunda (H.West ex Willd.) } \\
\text { O.Berg }\end{array}$ & 312 & 12,48 & 0,18 & 79,00 & 2,12 & 0,55 & 2,12 & 4,79 \\
\hline Psychotria vellosiana Benth. & 246 & 9,84 & 0,31 & 79,00 & 1,67 & 0,97 & 2,12 & 4,76 \\
\hline Ilex theezans Mart. ex Reissek & 251 & 10,04 & 0,24 & 80,00 & 1,71 & 0,73 & 2,15 & 4,59 \\
\hline$\cdots$ & $\ldots$ & $\ldots$ & $\ldots$ & $\ldots$ & $\ldots$ & $\ldots$ & $\ldots$ & $\ldots$ \\
\hline TOTAL & 14716 & 588,68 & 32,21 & 3721,00 & 100,00 & 100,00 & 100,00 & 300,00 \\
\hline
\end{tabular}

N: número de indivíduos; DA: densidade absoluta; DoA: dominância absoluta; FA: frequência absoluta; DR: densidade relativa; DoR: dominância relativa; FR: frequência relativa; IVI: índice de valor de importância. 

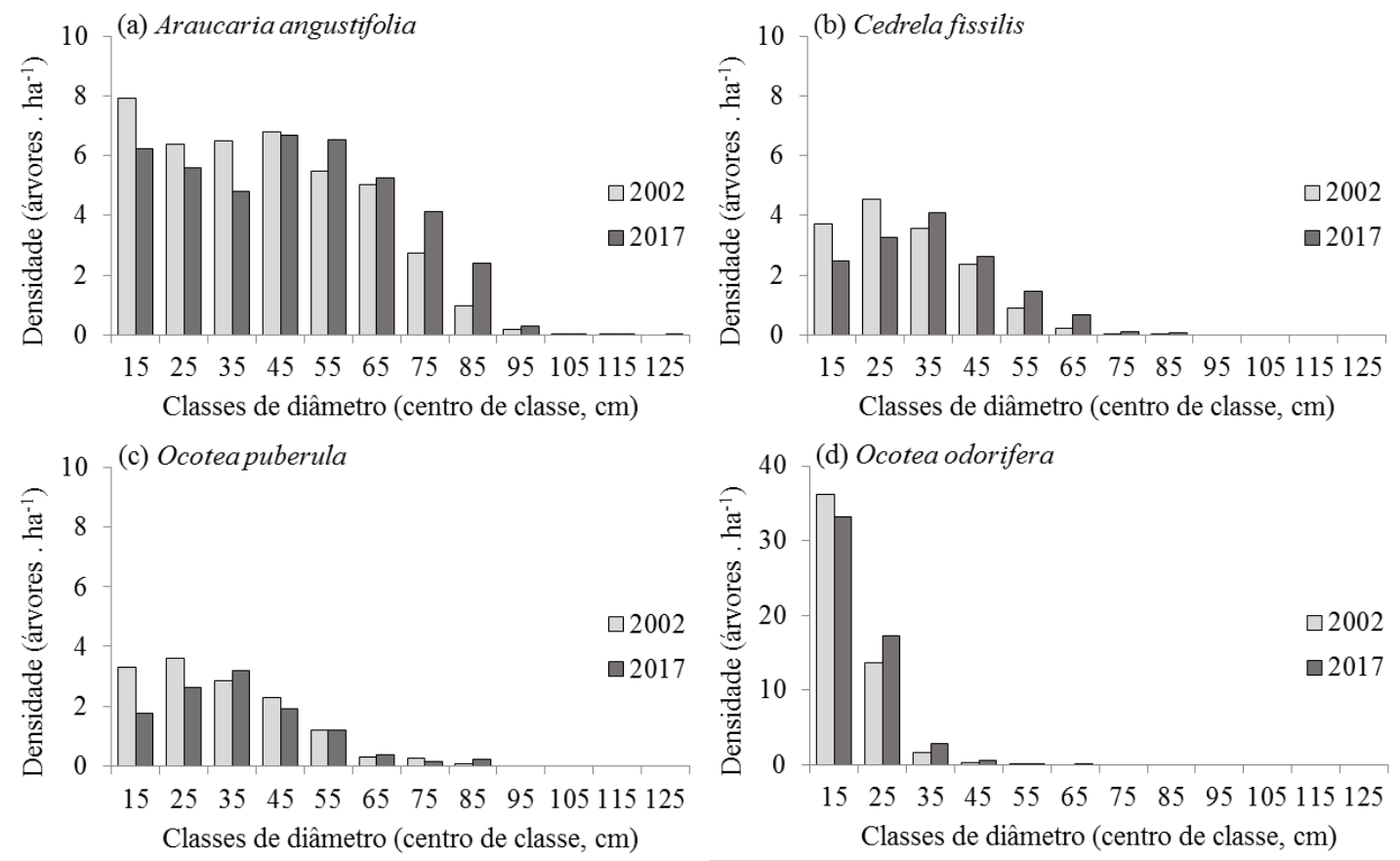

FIGURA 1 - Distribuição do número de árvores em classes diamétricas para as espécies A. angustifolia, C. fissilis, O. puberula e O. odorifera para os anos de 2002 e 2017. * Atenção para diferença de escala no eixo y de Ocotea odorifera.

\section{Índices de competição}

A relação de cada índice de competição testado com o incremento periódico em área transversal (IPAg) de cada espécie pode ser visualizada na Figura 2.

IC1

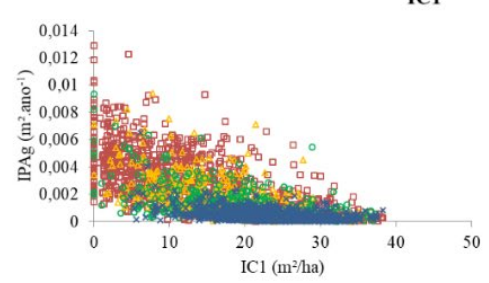

IC4

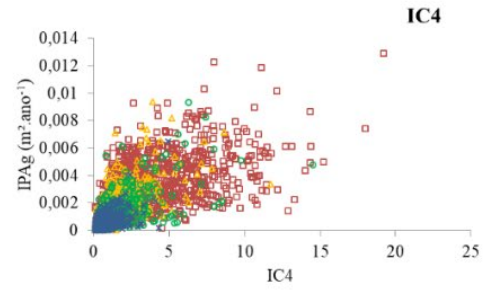

IC7

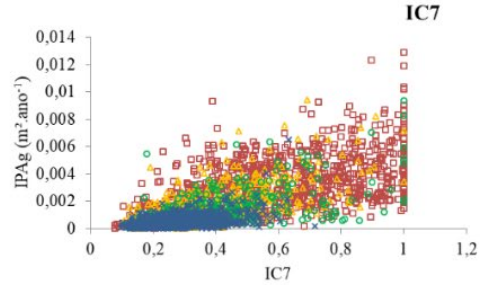

IC2

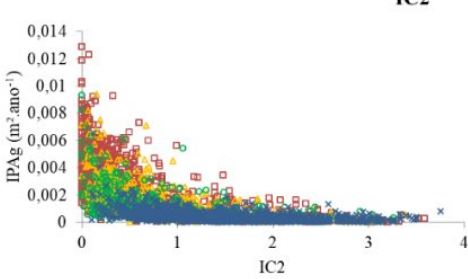

IC5

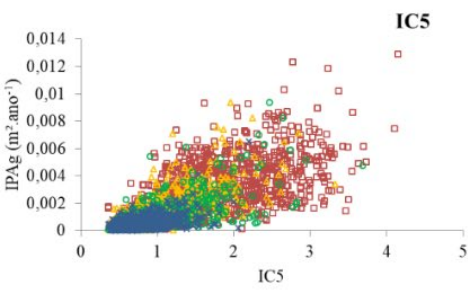

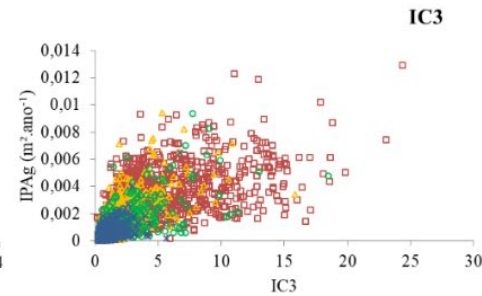

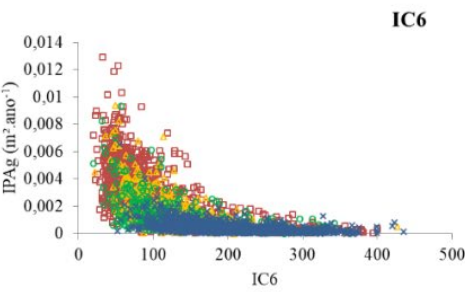

FIGURA 2 - Dispersão do incremento periódico anual em área transversal (IPAg) vs índices de competição (IC1, IC2, IC3, IC4, IC5, IC6 E IC7) para A. angustifolia, C. fissilis, O. puberula e O. odorifera em um fragmento de Floresta Ombrófila Mista. 
Analisando-se os valores encontrados para cada índice de competição, confirmam-se suas definições. Observa-se para os índices BAL (IC1 e IC2), que todas as espécies selecionadas, exceto $O$. odorifera, atingiram seu valor mínimo no zero, que neste caso estaria representando a ausência de competição unilateral. Isso indica que para as espécies $A$. angustifolia, C. fissilis e O. puberula, em pelo menos uma parcela, alguma árvore da espécie é a de maior diâmetro encontrada na comunidade florestal, o que não acontece em nenhum momento para 0 . odorifera. Para o índice IC7, a ausência de competição unilateral é representada pelo valor máximo de 1. Este valor ocorre, de forma análoga aos índices IC1 e IC2, quando a árvore de diâmetro máximo da parcela é a própria árvore objetivo. Coerentemente, o valor 1 também só foi alcançado pelas espécies $A$. angustifolia, $C$. fissilis e $O$. puberula.

Os índices IC3, IC4 e IC5 têm como característica alcançar valores muito próximo de 0 para representar alta competição, e podem alcançar diferentes valores, mais elevados, conforme a competição diminui. Na construção destes índices, o componente diâmetro da árvore objetivo no numerador e o diâmetro das competidoras no denominador é que traz essa característica. O índice IC6 apresentou variações em torno de valores mais elevados, uma vez que representa o somatório do diâmetro de cada competidora sobre o diâmetro da árvore $i$.

De modo geral, a competição que as árvores individuais da espécie Araucária estão submetidas foi menor, como esperado, que as demais angiospermas (Orellana \& Vanclay, 2018). Além disso, dentro do grupo das angiospermas também é possível verificar diferenciações, sendo que valores que representam maior competição foram encontrados para a espécie $O$. odorifera, espécie que é caracterizada segundo sua ecologia como nãopioneira (secundária tardia ou clímax) (Carvalho, 2005). Já C. fissilis e O. puberula, apresentaram valores intermediários e próximos entre si.

Árvores maiores, em geral, por alcançarem estratos superiores das florestas, têm menor competição (Cunha, 2009). Consequentemente, árvores de porte pequeno, localizadas no subbosque, estarão sob maiores influências competitivas. Isso foi observado quando se compara a distribuição diamétrica de cada espécie (Figura 1) e os respectivos valores dos índices de competição. As espécies com árvores de menores diâmetros apresentaram-se sob maior estresse competitivo, como é o caso de $O$. odorifera.

Referente a relação dos índices de competição e o IPAg, as variações observadas estão conexas aos distintos comportamentos que os índices expressam ao representar a competição natural. Para os índices IC1, IC2 e IC6, quanto menor o valor do índice, menor é a competição representada e maior será o crescimento. Alternadamente, para IC3, IC4, IC5 e IC8, menores valores do índice representam maior competição e, portanto, menor será o crescimento. Desta maneira, apesar das variações ligadas aos índices, a relação entre o crescimento (IPAg) e a competição foi caracterizada como inversa em todos os casos, ou seja, quanto mais elevada a competição, menor o crescimento e vice-versa. Este efeito negativo da competição sobre o crescimento foi confirmado por diversos autores (Holmes \& Reed, 1991; Contreras et al., 2011; Fraver et al., 2014; Tenzin et al., 2017).

Ademais, a maioria dos índices expressou esta relação com o crescimento de forma diferente da linear, aproximando-se mais de uma exponencial, em casos como o IC2 e IC6. Este comportamento também se aproxima, de certa maneira, da competição natural observada em uma floresta, ao entender-se que, sob diferentes níveis de competição, respostas na variação do crescimento em maior e menor proporção serão encontradas.

Na Tabela 3 são apresentados os coeficientes de correlação dos diferentes índices de competição e o incremento periódico anual em área transversal do período de 2002 a 2017. O logaritmo dos valores dos índices e do IPAg foi aplicado na tentativa de aproximar as relações da forma linear. Todos os índices testados foram significativamente correlacionados $(p<0,01)$. 
TABELA 3 - Correlação de Pearson entre o incremento periódico anual em área transversal (IPAg) e os índices de competição avaliados para todas as espécies (GERAL) e para cada espécie selecionada neste estudo em um fragmento de Floresta Ombrófila Mista.

\begin{tabular}{cccccc}
\hline \multirow{2}{*}{ Índice de competição } & \multicolumn{5}{c}{ Coeficiente de correlação } \\
& GERAL & A. angustifolia & C. fissilis & O. puberula & O. odorifera \\
IC1 & $-0,60$ & $-0,67$ & $-0,63$ & $-0,56$ & $-0,51$ \\
IC2 & $-0,71$ & $-0,77$ & $-0,76$ & $-0,67$ & $-0,65$ \\
IC3 & 0,74 & 0,84 & 0,83 & 0,73 & 0,67 \\
IC4 & 0,73 & 0,84 & 0,83 & 0,72 & 0,66 \\
IC5 & 0,74 & 0,84 & 0,83 & 0,72 & 0,67 \\
IC6 & $-0,75$ & $-0,84$ & $-0,81$ & $-0,72$ & $-0,68$ \\
IC7 & 0,70 & 0,80 & 0,77 & 0,69 & 0,60 \\
\hline
\end{tabular}

*Todos os valores foram significativos, $p<0,01$.

De forma análoga ao verificado na Figura 2, os valores negativos de correlação com o IPAg foram verificados para os índices IC1, IC2 e IC6, que representam a competição de maneira direta (menores valor, menor competição) e, portanto, o crescimento de maneira inversa.

De maneira geral, o comportamento de cada índice de competição foi semelhante entre o cômputo geral (comunidade florestal) e cada espécie, sendo que os índices IC3, IC4, IC5 e IC6 apresentaram valores muito próximos de correlação, destacando-se em relação aos demais. Além desses, o índice IC7 também aparece com bons resultados, cabendo ênfase devido a sua simplicidade de construção, uma vez que necessita apenas da medição da árvore de diâmetro máximo da parcela, além da árvore objetivo.

Apesar do menor desempenho no presente estudo, os índices IC1 e IC2 (Basal Area Larger) têm sido amplamente utilizados em modelos de árvores individuais (Monserud \& Sterba, 1996; Della-Flora, 2001; Yang et al., 2009; Lhotka \& Loewenstein, 2011; Orellana, 2014). Ressalta-se, portanto, a importância de avaliação de diferentes índices de competição para o contexto das modelagens, visto que, por não haver uma superioridade definida de um índice sobre outro, essa avaliação prévia pode auxiliar na obtenção de estimavas mais precisas e acuradas nas prognoses/projeções.

A espécie que teve seu crescimento melhor correlacionado com os índices de competição foi a Araucária, alcançando uma correlação de até 0,84 com o incremento em área transversal, para os índices IC3, IC4, IC5 e IC6. Chassot et al. (2011) avaliaram os índices IC1 e IC3, similares ao presente trabalho, por meio de sua correlação com o incremento diamétrico. Para eles, o valor mais acentuado de coeficiente de correlação foi encontrado para o índice IC1, observando valores não significativos para o índice IC3.

Mattos (2007) também empregou matriz de correlação para analisar a relação entre variáveis dendrométricas e do povoamento com os índices de IC1 e IC5, além de outros índices dependentes da distância, trabalhando com as espécies C. fissilis, Cabralea canjerana (Vell.) Mart. e Cordia trichotoma (Vell.) Arrab. ex Steud. Correlações entre os índices e o IPAg foram significativas e superiores para o índice IC5, similar ao presente trabalho, alcançado valores de 0,77 para C. fissilis. Esse valor de correlação foi superior inclusive aos dos índices que incluíram espacialidade em sua construção (dependentes da distância).

Além desses, vários outros autores utilizaram coeficiente de correlação de Pearson para selecionar índices de competição, como Silveira et al. (2015), estudando o crescimento de Trichilia claussenii C. DC em um fragmento de Floresta Semidecidual, RS e Castro et al. (2014), 
também uma Floresta Estacional Semidecidual, estudando a competição de uma grande quantidade de espécies.

Trabalhando com a espécie O. puberula, além de Nectandra megapotamica (Spreng.) Mez, Della-Flora (2001) formulou modelos de crescimento para as espécies em uma Floresta Estacional Decidual no Rio Grande do Sul, alcançando boas estimativas com a inclusão do índice IC1 como variável de competição.

\section{Análise da competição}

O índice IC6, por ter apresentado no geral maior coeficiente de correlação e pela boa relação gráfica com o IPAg, foi selecionado para modelar o crescimento (IPAg) em função da competição para cada uma das espécies. Os coeficientes estimados, o coeficiente de determinação $\left(R^{2}\right)$, o erro padrão da estimativa (Syx\%) e a significância da equação obtida para cada espécie e dos respectivos coeficientes são apresentados na Tabela 4.

TABELA 4 - Estimativa de coeficientes e estatísticas de ajuste e precisão do modelo de incremento periódico anual em área transversal (IPAg) em função do índice de competição de Lorimer (IC6) para cada uma das espécies deste estudo.

\begin{tabular}{|c|c|c|c|c|c|c|c|c|c|}
\hline Espécie & Co & ficientes & Std. Er. & Valor T & Prob. $>|t|$ & $\mathbf{R}^{2}$ & Syx(\%) & Valor F & Prob. $>F$ \\
\hline \multirow{2}{*}{ A. angustifolia } & $b_{0}$ & 0,009157 & 0,0004 & 25,14 & $<0,0001$ & \multirow{2}{*}{0,5458} & \multirow{2}{*}{49,30} & \multirow{2}{*}{1213,35} & \multirow{2}{*}{$<0,0001$} \\
\hline & $b_{1}$ & 0,985204 & 0,0007 & 1496,86 & $<0,0001$ & & & & \\
\hline \multirow{2}{*}{ C. fissilis } & $b_{0}$ & 0,011185 & 0,0008 & 14,01 & $<0,0001$ & \multirow{2}{*}{0,6226} & \multirow{2}{*}{49,19} & \multirow{2}{*}{625,71} & \multirow{2}{*}{$<0,0001$} \\
\hline & $b_{1}$ & 0,981325 & 0,0010 & 1005,96 & $<0,0001$ & & & & \\
\hline \multirow{2}{*}{ O. puberula } & $b_{0}$ & 0,008039 & 0,0007 & 10,78 & $<0,0001$ & \multirow{2}{*}{0,4774} & \multirow{2}{*}{64,41} & \multirow{2}{*}{298,59} & \multirow{2}{*}{$<0,0001$} \\
\hline & $b_{1}$ & 0,982869 & 0,0013 & 766,53 & $<0,0001$ & & & & \\
\hline \multirow{2}{*}{ O. odorifera } & $b_{0}$ & 0,003096 & 0,0002 & 19,11 & $<0,0001$ & \multirow{2}{*}{0,4837} & \multirow{2}{*}{63,63} & \multirow{2}{*}{1158,99} & \multirow{2}{*}{$<0,0001$} \\
\hline & $b_{1}$ & 0,988346 & 0,0004 & 2593,65 & $<0,0001$ & & & & \\
\hline
\end{tabular}

$b_{0}, b_{1}$ : coeficientes estimados; Std. Er.: erro padrão; R2: coeficiente de determinação; Syx\%: erro padrão da estimativa em porcentagem; Prob.>|t|: probabilidade de significância para o valor T; Prob.>F: probabilidade de significância para o valor F.

Utilizando um índice de competição de simples construção e independente da distância, quando ajustado por meio de técnicas de regressão, foi possível explicar até 55\%,62\%, 48\% e $48 \%$ da variação no crescimento periódico em área transversal nos últimos 15 anos das espécies $A$. angustifolia, C. fissilis, O. puberula e 0 . odorifera, respectivamente. Na Figura 3 são apresentados os valores de IPAg estimados para cada espécie em função do índice de competição IC6.

De acordo com os valores estimados e apresentados na Figura 3, fica clara a influência do fator espécie na relação crescimento-competição, verificando-se que alterações na competição podem refletir maiores ou menores variações no crescimento, dependendo da espécie, indicando haver espécies mais e menos sensíveis. Essas diferenças no nível de competição por espécies podem ser associadas às diferenças na tolerância à sombra que cada uma apresenta (Tenzin et al., 2017).

Em níveis de mais baixa competição, maior IPAg é evidente para A. angustifolia, C. fissilis e $O$. puberula. No entanto, a espécie $O$. odorifera parece ser menos sensível às mudanças nas condições de luz, pois o aumento da competição para esta espécie revela uma diminuição menos abrupta do IPAg como resposta, diferentemente do que é esperado para $C$. fissilis, $A$. angustifolia e $O$. puberula (Figura 3). Confrontando-se estes resultados com as características ecológicas das espécies, tanto $A$. angustifolia quanto $C$. fissilis são espécies consideradas exigentes em luz (Carvalho, 2003), enquanto O. puberula pode ser considerada semi-heliófila (Ortega, 1995). Alternadamente, O. odorifera é um espécie tolerante à sombra (Carvalho, 
2005). Sob essa avaliação, os resultados encontrados são muito coerentes e apresentam consistência com sugestões anteriores de Buffum et al. (2008); Tenzin \& Hasenauer (2016) e Tenzin et al. (2017). Além disso, esta observação também se firma com relação aos grupos ecológicos que essas espécies representam.

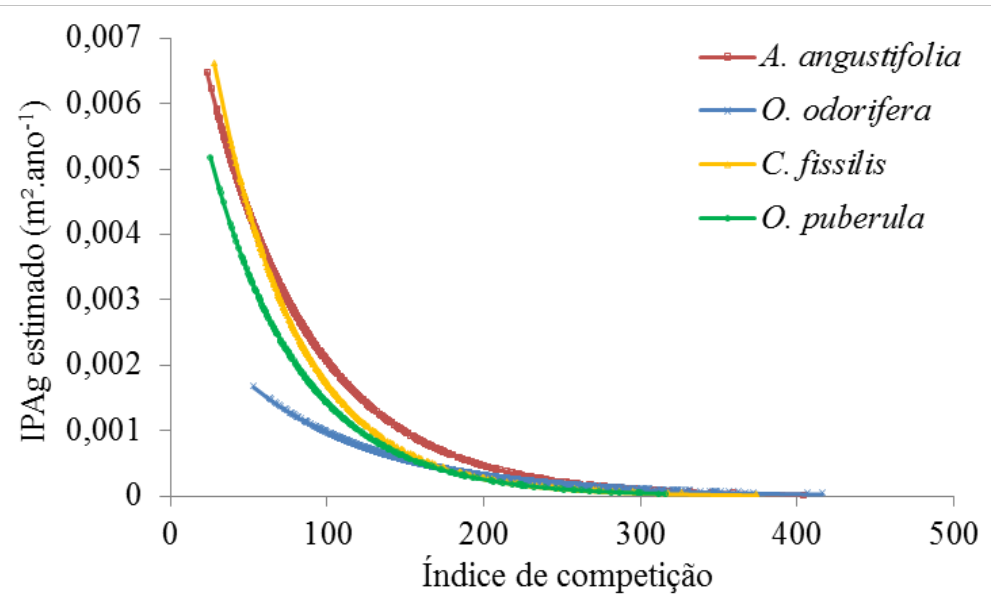

FIGURA 3 - Incremento periódico anual em área transversal (IPAg) estimado $\left(\mathrm{m}^{2}\right.$.ano-1) vs índice de competição de Lorimer (IC6) para cada uma das espécies selecionadas no fragmento em estudo.

Apesar de que resultados coerentes na análise entre espécies tenham sido observados, deve-se ponderar pelo fato de que, dentro de uma mesma parcela, em fustes de mesmo porte são atribuídos índices de competição com valores muito próximos, mesmo que pertençam a espécies diferentes e que não possuam as mesmas taxas de crescimento. Por esta razão é possível que variáveis adicionais, especialmente relacionadas à copa, pudessem contribuir para análises das estratégias competitivas das espécies e de cada indivíduo (Canham et al., 2004; Coates et al., 2009).

Além da variação observada entre espécies, tem-se que considerar que a competição é um processo dinâmico. Perturbações na floresta, por exemplo, que são mecanismos para iniciar a sucessão, têm efeitos a longo prazo, e alteram a dinâmica competitiva entre as árvores (Weber et al., 2008). Por isso, variações na competição ao longo do tempo também devem ser levadas em conta tendo em vista projeções de crescimento.

Posto isto, este trabalho contribui com subsídios para a inclusão de índices de competição em submodelos de incremento no contexto da modelagem em nível de árvore individual em florestas inequiâneas. Como a competição não é uma variável de medição direta, a possibilidade de representar a competição natural em uma floresta por meio de índices auxilia a expressar o estresse competitivo que cada árvore está sujeita, contribuindo, por sua vez, para projeções de crescimento.

\section{CONCLUSÕES}

- Os índices de competição independentes da distância testados são recomendados para representar a competição das espécies estudadas em Floresta Ombrófila Mista, tendo sido encontradas correlações significativas entre estes e o incremento periódico em área transversal das árvores no fragmento, com destaque para o índice de Lorimer (IC6).

- A relação competição-crescimento apresentou diferenças entre as espécies avaliadas, sugerindo espécies com crescimento mais e menos sensíveis à variação da competição.

\section{REFERÊNCIAS}

Alvares, C. A., Stape, J. L., Sentelhas, P. C., Gonçalves, J. L. M., \& Sparovek, G. (2013). Köppen's climate classification map for Brazil. Meteorologische Zeitschrift, 22(6), 711-728.

http://dx.doi.org/10.1127/0941-2948/2013/0507. 
Arce, J. E., Koehler, A., Jaster, C. B., \& Sanquetta, C. R. (2002). Florexel: funções florestais desenvolvidas para o Microsoft Exce/@. Curitiba: Centro de Ciências Florestais e da Madeira, Universidade Federal do Paraná.

Begon, M., Townsend, C. R., \& Harper, J. L. (2007). Ecologia de indivíduos a ecossistemas (4. ed.). Porto Alegre: Artmed.

Buffum, B., Gratzer, G., \& Tenzin, Y. (2008). The sustainability of selection cutting in a late successional broadleaved community forest in Bhutan. Forest Ecology and Management, 256(12), 2084-2091. http://dx.doi.org/10.1016/j.foreco.2008.07.031.

Burkhart, H. E., \& Tomé, M. (2012). Modeling forest trees and stands. Dordrecht: Springer. http://dx.doi.org/10.1007/978-90-481-3170-9.

Canham, C. D., Lepage, P. T., \& Coates, K. D. (2004). A neighborhood analysis of canopy tree competition: effects of shading versus crowding. Canadian Journal of Forest Research, 34(4), 778-787. http://dx.doi.org/10.1139/x03-232.

Carvalho, P. E. R. (2003). Espécies arbóreas brasileiras (1. ed., Vol. 1). Brasília: Embrapa Informação Tecnológica.

Carvalho, P. E. R. (2005). Canela-sassafrás (Circular Técnica, No. 110). Colombo: Embrapa Florestas. Recuperado em 16 de setembro de 2018, de http://www.infoteca.cnptia.embrapa.br/handle/doc/288823

Castagneri, D., Vacchiano, G., Lingua, E., \& Motta, R. (2008). Analysis of intraspecific competition in two subalpine Norway spruce (Picea abies (L.) Karst.) stands in Paneveggio (Trento, Italy). Forest Ecology and Management, 255(3-4), 651-659. http://dx.doi.org/10.1016/j.foreco.2007.09.041.

Castro, R. V. O., Soares, C., Leite, H., Souza, A., Martins, F., Nogueira, G., Oliveira, M., \& Silva, F. (2014). Competição em nível de árvore individual em uma Floresta Estacional Semidecidual. Silva Lusitana, 22(1), 43-66.

Chassot, T., Fleig, F. D., Finger, C. A. G., \& Longhi, S. J. (2011). Modelos de crescimento em diâmetro de árvores individuais de Araucaria angustifolia (Bertol.) Kuntze em Floresta Ombrófila Mista. Ciência Florestal, 21(2), 303-313. http://dx.doi.org/10.5902/198050983234.

Coates, K. D., Canham, C. D., \& Lepage, C. T. (2009). Above versus below ground competitive effects and responses of a guild of temperate tree species. Journal of Ecology, 97(1), 118-130. http://dx.doi.org/10.1111/j.1365-2745.2008.01458.x.

Contreras, M. A., Affleck, D., \& Chung, W. (2011). Evaluating tree competition indices as predictors of basal area increment in western Montana forests. Forest Ecology and Management, 262(11), 19391949. http://dx.doi.org/10.1016/j.foreco.2011.08.031.

Cunha, T. A. (2009). Modelagem do incremento de arvores individuais de Cedrela odorata L. na Floresta Amazônica (Dissertação de mestrado). Universidade Federal de Santa Maria, Santa Maria.

Curto, R. A. (2015). Avaliação do crescimento e potencial de manejo em plantio superestocado de Araucaria angustifolia (Bert.) O. Ktze (Tese de doutorado). Setor de Ciências Agrárias, Universidade Federal do Paraná, Curitiba.

Daniels, R. F., Burkhart, H. E., \& Clason, T. R. (1986). A comparison of competition measures for predicting growth of loblolly pine trees. Canadian Journal of Forest Research, 16(6), 1230-1237. http://dx.doi.org/10.1139/x86-218.

Della-Flora, J. B. (2001). Modelos de crescimento para árvores singulares - Nectandra megapotamica (Spreng.) Mez. e Ocotea puberula Ness (Dissertação de mestrado). Universidade Federal de Santa Maria, Santa Maria.

Ebling, Â. A., \& Péllico Netto, S. (2015). Modelagem de ocorrência de coortes na estrutura diamétrica da araucaria angustifolia (Bertol.) Kuntze. Cerne, 21(2), 251-257. http://dx.doi.org/10.1590/01047760201521111667.

Figueiredo Filho, A., Dias, A. N., Stepka, T. F., \& Sawczuk, A. R. (2010). Crescimento, mortalidade, ingresso e distribuição diamétrica em floresta ombrófila mista. Floresta, 40(4), 763-776. http://dx.doi.org/10.5380/rf.v40i4.20328.

Fraver, S., D'Amato, A. W., Bradford, J. B., Jonsson, B. G., Jönsson, M., \& Esseen, P.-A. (2014). Tree growth and competition in an old-growth Picea abies forest ofboreal Sweden: influence of tree spatial patterning. Journal of Vegetation Science, 25(2), 374-385. http://dx.doi.org/10.1111/jvs.12096.

Glover, G. R., \& Hool, J. N. (1979). A basal area ratio predictor of loblolly pine plantation mortality. Forest Science, 25(2), 275-282. 
Holmes, M. J., \& Reed, D. D. (1991). Competition indices for mixed species northern hardwoods. Forest Science, 37(5), 1338-1349.

Lhotka, J. M., \& Loewenstein, E. F. (2011). An individual-tree diameter growth model for managed uneven-aged oak-shortleaf pine stands in the Ozark Highlands of Missouri, USA. Forest Ecology and Management, 261(3), 770-778. http://dx.doi.org/10.1016/j.foreco.2010.12.008.

Lorimer, C. G. (1983). Test of age-independent competition indices for individual trees in natural hardwood stands. Forest Ecology and Management, 6(4), 343-360. http://dx.doi.org/10.1016/03781127(83)90042-7.

Mattos, R. B. (2007). Produtividade e incremento de Cabralea canjerana (Vell.) Mart., Cedrela fissilis Cell. $e$ Cordia trichotoma (Vell.) Arrab. ex steud., em floresta nativa no Rio Grande do Sul (Tese de doutorado). Universidade Federal de Santa Maria, Santa Maria.

Monserud, R. A., \& Sterba, H. (1996). A basal area increment model for individual trees growing in evenand uneven-aged forest stands in Austria. Forest Ecology and Management, 80(1-3), 57-80. http://dx.doi.org/10.1016/0378-1127(95)03638-5.

Munro, D. D. (1974). Forest growth model: a prognosis. In J. Fries (Ed.), Growth models for tree and stand simulation (Research Notes, No. 30, pp. 7-21). Stockholm: Royal College of Forestry.

Orellana, E. (2014). Sucessão florestal, regimes de manejo e competição avaliados por modelos de árvore individual em um fragmento de Floresta de Araucária (Tese de doutorado). Setor de Ciências Agrárias, Universidade Federal do Paraná, Curitiba.

Orellana, E., \& Vanclay, J. K. (2018). Competition and dominance between angiosperms and Araucaria angustifolia (Bert.) O. Kuntze in the Atlantic Forest in southern Brazil. Forest Ecology and Management, 425, 119-125. http://dx.doi.org/10.1016/j.foreco.2018.05.022.

Ortega, L. S. (1995). Temperamento de luz de los árboles del alto Paraná y potencial de regeneración forestal. Ka'aguy Revista Forestal de Paraguay , 11(1), 16-20.

Peet, R. K., \& Christensen, N. L. (1987). Competition and tree death. Bioscience, 37(8), 586-595. http://dx.doi.org/10.2307/1310669.

Peltoniemi, M., \& Mäkipää, R. (2011). Quantifying distance-independent tree competition for predicting Norway spruce mortality in unmanaged forests. Forest Ecology and Management, 261(1), 30-42. http://dx.doi.org/10.1016/j.foreco.2010.09.019.

Ryder, N. B. (1965). The Cohort as a concept in the study of social change. American Sociological Review, 30(6), 843-861. PMid:5846306. http://dx.doi.org/10.2307/2090964.

Silveira, B. D., Floriano, E. P., Nakajima, N. Y., Hosokawa, R. T., Rosot, N. C., \& Gracioli, C. R. (2015). Relação da morfometria e competição com o crescimento de Trichilia claussenii em um fragmento de Floresta Semidecidual, RS. Floresta, 45(2), 373-382. http://dx.doi.org/10.5380/rf.v45i2.35164.

Stage, A. R. (1973). Prognosis model for stand development (Research Paper, No. INT-137). Madison: USDA Forest Service. http://dx.doi.org/10.5962/bhl.title.69018.

Tenzin, J., \& Hasenauer, H. (2016). Tree species composition and diversity in relation to anthropogenic disturbances in broad-leaved forests of Bhutan. The International Journal of Biodiversity Science, Ecosystem Services \& Management, 12, 274-290. http://dx.doi.org/10.1080/21513732.2016.1206038.

Tenzin, J., Tenzin, K., \& Hasenauer, H. (2017). Individual tree basal area increment models for broadleaved forests in Bhutan. Forestry, 90, 367-380. http://dx.doi.org/10.1093/forestry/cpw065.

Téo, S. J., Figueiredo Filho, A., \& Lingnau, C. (2015). Análise espacial do estresse competitivo, incremento diamétrico e estrutura de uma Floresta Ombrófila Mista, Irati, PR. Floresta, 45(4), 681-694. http://dx.doi.org/10.5380/rf.v45i4.39939.

Tomé, M., \& Burkhart, H. E. (1989). Distance-dependent competition measures for predicting growth of individual tree. Forest Science, 35(3), 816-831.

Tonini, H. (2007). Índices de competição e o seu uso na modelagem do crescimento das árvores (Documentos, No. 8). Boa Vista: Embrapa Roraima.

Weber, P., Bugmann, H., Fonti, P., \& Rigling, A. (2008). Using a retrospective dynamic competition index to reconstruct forest succession. Forest Ecology and Management, 254(1), 96-106. http://dx.doi.org/10.1016/j.foreco.2007.07.031.

Weiskittel, A. R., Hann, D. W., Kershaw, J. A., \& Vanclay, J. K. (2011). Forest growth and yield modeling. Oxford: Wiley-Blackwell. http://dx.doi.org/10.1002/9781119998518.

Yang, Y., Huang, S., Meng, S. X., Trincado, G., \& Vanderschaaf, C. L. (2009). A multilevel individual tree basal area increment model for aspen in boreal mixedwood stands. Canadian Journal of Forest Research, 39(11), 2203-2214. http://dx.doi.org/10.1139/X09-123. 
Zhao, D., Borders, B., \& Wilson, M. (2004). Individual-tree diameter growth and mortality models for bottomland mixed-species hardwood stands in the lower Mississippi alluvial valley. Forest Ecology and Management, 199(2-3), 307-322. http://dx.doi.org/10.1016/j.foreco.2004.05.043.

Contribuição dos autores: CTS: conceituação, curadoria de dados, análise formal, investigação, metodologia, administração do projeto, escrita - primeira redação, escrita - revisão e edição, AFF: conceituação, investigação, metodologia, administração do projeto, supervisão, escrita - revisão e edição, TLS: investigação, supervisão, visualização, escrita - revisão e edição, RGMN: conceituação, investigação, metodologia, escrita - revisão e edição. 